\title{
The Artificial Compression Method for Computation of Shocks and Contact Discontinuities: III. Self-Adjusting Hybrid Schemes
}

\author{
By Amiram Harten
}

\begin{abstract}
This paper presents a new computational method for the calculation of discontinuous solutions of hyperbolic systems of conservation laws, which deal effectively with both shock and contact discontinuities. The method consists of two stages: in the first stage a standard finite-difference scheme is hybridized with a nonoscillatory first order accurate method to provide for the monotonic variation of the solution near discontinuities, and in the second stage artificial compression is applied to sharpen transitions at discontinuities. This modification of a standard finite-difference method results in a scheme which preserves the order of truncation error of the original method and yet yields a sharp and oscillation free transition for both shocks and contact discontinuities. The modification can be easily implemented in existing computer codes.
\end{abstract}

1. Introduction. The capturing approach to the calculation of discontinuous solutions of hyperbolic systems of conservation laws by finite-difference methods has two essential defects: 1. A jump discontinuity in the solution of the partial differential equation is approximated in the solution of a finite-difference scheme by a continuous transition connecting the states on both sides of the jump discontinuity. When this jump discontinuity is an admissible shock, the transition occurs within a finite number of mesh points (typically 4-10, depending on the scheme and the strength of the shock). However, if the jump discontinuity is a contact discontinuity, then the width of the transition is typically unbounded in time; it behaves like $n^{1 /(R+1)}$ where $n$ is the number of time steps taken and $R$ is the order of accuracy of the finite-difference scheme. As a result of the smearing of the discontinuity in the numerical solution one has to use a mesh finer than is actually needed in smooth regions of the solution. However, this causes computational difficulties, especially in multidimensional calculations. 2. The transition in the finite-difference approximation to a jump discontinuity is not always monotonic. Finite-difference schemes, in particular those with order of accuracy larger than one, may produce overshoots or undershoots when applied across a discontinuity. These oscillations not only damage the accuracy of the numerical solution, but can also induce nonlinear instabilities (see [1]), and trigger convergence to nonphysical solutions (see [11]).

Following the pioneering work of von Neumann and Richtmyer [27], many other viscosity type methods have been developed (e.g. see [17], [15], [12], [13]). These methods are capable of suppressing the post shock oscillations, but at the expense of introducing extra smearing of the discontinuity. 
Methods to reduce the spread in the finite-difference approximation to discontinuities have been developed only recently. Examples are the antidiffusion (or flux corrected transport) method of Boris and Book [1], Chorin's implementation of the Glimm scheme [3], and the artificial compression method (ACM) of the author [8]. Discontinuities computed with these methods are very sharp and oscillation-free; however, these schemes are only first order accurate.

In this paper, the third in a series on the ACM, we describe how to combine the idea of the ACM with that of the self-adjusting hybrid schemes [13], to obtain oscillation-free sharp transitions of discontinuities while maintaining high order of truncation error in the smooth part of the solution. In Section 2 we share our ignorance of monotonicity with the reader. The first order accurate ACM is described in detail and analyzed in [9] and [10]; for the convenience of the reader we review it briefly in Section 3. In Section 4 we discuss hybridization and automatic switches. In Section 5 we describe the combined hybrid ACM. In Section 6, we examine the reasons for implementing the hybrid ACM idea. We conclude the paper with a summary.

2. Monotonicity of Finite-Difference Schemes. We consider finite-difference solutions to hyperbolic systems of conservation laws

$$
w_{t}+f(w)_{x}=0,
$$

where $w=\left(w^{1}, \ldots, w^{M}\right)^{T}, f(w)=\left(f^{1}(w), \ldots, f^{M}(w)\right)^{T}$ and the matrix

$$
A(w)=\operatorname{grad}_{w} f
$$

has $M$ real and distinct eigenvalues $a_{1}(w), \ldots, a_{M}(w)$. We say that the finite-difference operator

$$
w_{j}=(L v)_{j}=H\left(v_{j-k}, v_{j-k+1}, \ldots, v_{j+k}\right)
$$

is in conservation form (or conservative) if the function $H$ can be written as

$$
H\left(v_{j-k}, \ldots, v_{j+k}\right)=v_{j}-\lambda\left(h_{j+1 / 2}-h_{j-1 / 2}\right),
$$

where

$$
h_{j+1 / 2}=h\left(v_{j-k+1}, \ldots, v_{j+k}\right), \quad h_{j-1 / 2}=h\left(v_{j-k}, \ldots, v_{j+k-1}\right)
$$

the consistency condition on the numerical flux $h$ is

$$
h(w, w, \ldots, w)=f(w),
$$

$\lambda=\Delta t / \Delta x$, where $\Delta t$ and $\Delta x$ are the time and space increments, respectively. The conservation form is important since it guarantees that the computed discontinuities propagate with the correct speed (see [17]).

It is of particular computational interest to derive conditions under which a finitedifference scheme does not generate oscillations upon crossing a discontinuity; such schemes are a posteriori termed nonoscillatory schemes. We say that the finite-difference operator (2.2) is monotone (or positive) if

$$
H_{l} \equiv \frac{\partial H}{\partial v_{j+l}}\left(v_{j-k}, v_{j-k+1}, \ldots, v_{j+k}\right) \geqslant 0, \quad-k \leqslant l \leqslant k .
$$


In the matrix case inequality (2.4) is taken to mean that the eigenvalues of $H_{l}$ are nonnegative. It was shown in [11] that monotone schemes are of first order accuracy; the leading term in the truncation error is of second order. The solution of the difference equation satisfies, modulo smaller truncation error, the parabolic equation

$$
\begin{gathered}
w_{t}+f(w)_{x}=\Delta t\left[B(w, \lambda) w_{x}\right]_{x}, \\
B(w, \lambda)=\frac{1}{2 \lambda^{2}}\left[\sum_{l=-k}^{k} l^{2} H_{l}(w, w, \ldots, w)-\lambda^{2} A^{2}(w)\right], \\
B(w, \lambda) \geqslant 0, \quad B(w, \lambda) \neq 0 .
\end{gathered}
$$

Equation (2.5a) is called the modified equation (see [28]).

In the scalar case $(M=1)$ a monotone finite-difference operator $L,(2.2)$ and (2.4), is monotonicity preserving, i.e. its operation, $L V$, on any monotonic mesh function $v$, is also monotonic and in the same sense (see [5]). Hence, monotone schemes are nonoscillatory. In the linear case $f(w)=A w, A=$ constant,

$$
H\left(v_{j-k}, v_{j-k+1}, \ldots, v_{j+k}\right)=\sum_{l=-k}^{k} C_{l} v_{j+l}
$$

with

$$
\sum_{l=-k}^{k} C_{l}=I, \quad \sum_{l=-k}^{k} l C_{l}=\lambda A, \quad C_{l}=\text { constant }
$$

A scheme is monotone if and only if

$$
C_{l} \geqslant 0, \quad-k \leqslant l \leqslant k
$$

When the coefficients $C_{l}$ are simultaneously diagonalized the scheme (2.6) separates into $M$ decoupled scalar schemes for the Riemann invariants; these scalar schemes are monotonicity preserving. In the general case of nonlinear systems we have not as yet succeeded in proving that monotone schemes are nonoscillatory; however, there is enough numerical evidence to support such a conjecture.

We remark that, in the linear scalar case (2.6) monotonicity of a scheme is a necessary and sufficient condition for it to be oscillation-free (see [5]); in general monotonicity is sufficient but not necessary (see [9]). Even in the case of a scalar linear flux, $f=$ constant. $w$, a nonlinear scheme, can be monotonicity preserving without being monotone. In fact, in Section 4 of this paper we construct a monotonicity preserving scheme which is second order accurate (see also [26]).

Next we derive a necessary condition for a scheme to be monotonicity preserving. Subtract (2.2) at $j=i$ from (2.2) at $j=i+1$ and use the mean value theorem to obtain 
$w_{i+1}-w_{i}=H\left(v_{i-k+1}, \ldots, v_{i+k+1}\right)-H\left(v_{i-k}, \ldots, v_{i+k}\right)$

$$
\begin{aligned}
= & \sum_{l=-k}^{k} H_{l}\left(v_{i-k}+\eta\left(v_{i-k+1}-v_{i-k}\right), \ldots, v_{i+k}+\eta\left(v_{i+k+1}-v_{i+k}\right)\right) \\
& \cdot\left(v_{i+l+1}-v_{i+l}\right), \quad 0 \leqslant \eta \leqslant 1 .
\end{aligned}
$$

Choosing $v_{i-k}=\cdots=v_{i+l}=v_{L}, v_{i+l+1}=\cdots=v_{i+k+1}=v_{k},-k \leqslant l \leqslant k,(2.7 \mathrm{a})$ becomes

$$
w_{i+1}-w_{i}=H_{l}\left(v_{L}, \ldots, v_{L}+\left(v_{R}-v_{L}\right), v_{R}, \ldots\right)\left(v_{R}-v_{L}\right) .
$$

Hence a necessary condition for (2.2) to be monotonicity preserving is

$$
H_{l}\left(v_{L}, \ldots, v_{L}+\eta\left(v_{R}-v_{L}\right), v_{R}, \ldots\right) \geqslant 0
$$

for all $-k \leqslant l \leqslant k$ and $0 \leqslant \eta \leqslant 1$.

Condition (2.8) will be used in Section 4 in order to derive nonoscillatory schemes. A necessary and sufficient condition to characterize nonoscillatory schemes is not yet available.

\section{First Order Accurate ACM. Let}

$$
w_{t}+f(w)_{x}=0
$$

be a scalar conservation law. Denote by $\left(w_{L}, w_{R}, S\right)$ the progressive wave solution to (3.1),

$$
\begin{gathered}
w(x, t)= \begin{cases}w_{L}, & x<S t, \\
w_{R}, & x>S t,\end{cases} \\
f\left(w_{R}\right)-f\left(w_{L}\right)=S\left(w_{R}-w_{L}\right),
\end{gathered}
$$

where (3.2b) is the Rankine-Hugoniot ( $\mathrm{RH})$ relation. Let

$$
s\left(w, w_{L}\right)=\left[f(w)-f\left(w_{L}\right)\right] /\left(w-w_{L}\right) ;
$$

note that $s\left(w_{L}, w_{R}\right)=S$. A progressive wave solution $\left(w_{L}, w_{R}, S\right)$ is admissible (or physical) if it satisfies Oleinik's entropy condition (see [21])

$$
s\left(w, w_{L}\right) \geqslant S
$$

for all $w$ between $w_{L}$ and $w_{R}$. If (3.2c) is a strict inequality, then $\left(w_{L}, w_{R}, S\right)$ is a shock; if in (3.2c) equality holds for all $w$ between $w_{L}$ and $w_{R}$, then $\left(w_{L}, w_{R}, S\right)$ is a contact discontinuity.

Jennings [14] showed that if $\left(w_{L}, w_{R}, S\right)$ is a shock, then every monotone scheme in conservation form possesses a steady progressing monotonic profile (discrete shocks). The number of cells $W\left(w_{-}, w_{+}\right)$, occupied by values lying between $w_{-}$and $w_{+}$in the profile, is approximately equal to the width of the viscous profile of the modified equation (2.5) which is given by

$$
W\left(w_{-}, w_{+}\right)=\lambda \int_{w_{-}}^{w_{+}} \frac{B(w, \lambda) d w}{\left[s\left(w, w_{L}\right)-S\right]\left(w-w_{L}\right)},
$$


where $s\left(w, w_{L}\right)$ is given by (3.2c). If $\left(w_{L}, w_{R}, S\right)$ is a contact discontinuity, i.e. $s\left(w, w_{L}\right)$ $\equiv S$ for all $w$ between $w_{L}$ and $w_{R}$, then linear finite-difference schemes do not possess a steady progressing solution, i.e. $W \rightarrow \infty$ as $n \rightarrow \infty$.

The artificial compression method (ACM) is motivated by the following observation: let $g(w)$ be an artificial compression flux (ACF) defined by

$$
g(w)=\alpha(w)\left(w-w_{L}\right)
$$

where

$$
\alpha\left(w_{R}\right)=0 ; \quad \alpha(w)>0 \text { for all } w \text { between } w_{L} \text { and } w_{R}
$$

Then, if $\left(w_{L}, w_{R}, S\right)$ is a contact discontinuity or a shock of the original conservation law (3.1), it is a shock of the equation

$$
w_{t}+[f(w)+g(w)]_{x}=0 .
$$

The essence of the ACM is to compute admissible discontinuities of the original conservation law (3.1) as solutions of an equation of the type (3.4c), i.e., to modify a nonoscillatory scheme $L$ for the solution of (3.1) by replacing the flux $f$ in $L$ by $f+g$. Denote the modified scheme by $\hat{L}$. Note that increasing $g(w)$ in (3.4) increases the denominator in (3.3), thereby improving the resolution of the computed discontinuity. Even if $\left(w_{L}, w_{R}, S\right)$ is a contact discontinuity, its numerical approximation is a nonsmearing profile.

It is convenient to separate the artificial compression from the main calculation by operator splitting. Let $\hat{L}=C L$, with $L$ a finite-difference approximation to the solution operator of the original conservation law (3.1) and $C$ some finite-difference approximation to the solution operator of the equation

$$
w_{t}+g(w)_{x}=0 \text {. }
$$

Note that if $\left(w_{L}, w_{R}, S\right)$ is an admissible solution of $(3.1)$, then $\left(w_{L}, w_{R}, 0\right)$ is a stationary shock of (3.5). The artificial compression operator is denoted by $C$.

We choose $C$ to be the one-sided scheme

$$
\begin{gathered}
w_{j}^{n+1}=w_{j}^{n}-(\hat{\lambda} / 2)\left(G_{j+1 / 2}^{n}-G_{j-1 / 2}^{n}\right), \\
G_{j+1 / 2}^{n}=g_{j}^{n}+g_{j+1}^{n}-\left|g_{j+1}^{n}-g_{j}^{n}\right| \operatorname{sgn}\left(w_{j+1}^{n}-w_{j}^{n}\right) ;
\end{gathered}
$$

here $g_{j}^{n}=g\left(w_{j}^{n}\right)$. The one-sided scheme is monotonicity preserving under the discrete Courant-Friedrichs-Lewy (CFL) condition

$$
\hat{\lambda} \max _{w_{j} \neq w_{j+1}}\left|\frac{g_{j+1}-g_{j}}{w_{j+1}-w_{j}}\right| \leqslant 1,
$$

and it is capable of producing maximal resolution of stationary shocks.

We have dealt, for purposes of analysis, with profiles of admissible discontinuities. $w_{L}$ and $w_{R}$ used in the construction of the ACF $g$ in (3.4) are in general not known in advance. Nevertheless, we can construct a mesh function $g^{n}$, which has properties similar to (3.4a) and (3.4b), by extracting the required information directly from the 
numerical solution $w^{n}$ (see [9]). Define $g_{j}^{n}$ by

$$
\begin{aligned}
g_{j}^{n}= & \operatorname{sgn}\left(w_{j+1}^{n}-w_{j}^{n}\right) \\
& \cdot \max \left\{0, \min \left[\left|w_{j+1}^{n}-w_{j}^{n}\right|,\left(w_{j}^{n}-w_{j-1}^{n}\right) \operatorname{sgn}\left(w_{j+1}^{n}-w_{j}^{n}\right)\right]\right\}
\end{aligned}
$$

It follows directly from the definition (3.7a) that

$$
\left|g_{j+1}-g_{j}\right| \leqslant\left|w_{j+1}-w_{j}\right|
$$

thus, as a consequence, the CFL condition (3.6c) is satisfied when $\hat{\lambda} \leqslant 1$. The artificial compression operator $C_{\Delta}$, is the one-sided scheme (3.6) with $g$ defined by (3.7a); $C_{\Delta}$ is called an artificial compression operator since successive applications of $C_{\Delta}$ to initial data of the form

$$
w_{j}^{0}= \begin{cases}u_{L}, & j \leqslant J_{L}, \\ u_{j}, & J_{L} \leqslant j \leqslant J_{R}, \\ u_{R}, & J_{R} \leqslant j\end{cases}
$$

where $u_{J_{L}}=u_{L}, u_{J_{R}}=u_{R}$ and $u$ is a strictly monotone function of $j$, converges pointwise to the stationary shock-like solution

$$
w_{j}^{\infty}= \begin{cases}u_{L}, & j<J_{\infty}, \\ u_{L}+\eta\left(u_{R}-u_{L}\right), & j=J_{\infty}, \\ u_{R}, & j>J_{\infty} .\end{cases}
$$

Here $0 \leqslant \eta \leqslant 1, J_{R} \geqslant J_{\infty} \geqslant J_{L}$. When $C_{\Delta}$ is applied in a region where $w_{x x}$ is bounded

$$
C_{\Delta} w=w+O\left((\Delta x)^{2}\right) \text {. }
$$

Equation (3.9) is not true where $w$ or $w_{x}$ is discontinuous; consequently, one should not apply $C_{\Delta}$ to an inadmissible discontinuity or to end points of a rarefaction wave.

We now turn to a description of the ACM for a system of conservation laws. As in (2.1) $w$ corresponds to an $M$-vector $w=\left(w_{1}, \ldots, w_{M}\right)^{T}$, and $f(w)$ is the vector function $f(w)=\left(f_{1}(w), \ldots, f_{M}(w)\right)^{T}$. The matrix $A=\operatorname{grad}_{w} f$ has $M$ real and distinct eigenvalues $a_{1}, \ldots, a_{M}$; we denote by $r_{1}, \ldots, r_{M}$ the corresponding right eigenvectors of $A$ and by $S_{L}$ the shock set of $w_{L}$, i.e. all states $w$ that satisfy the RH relation with $w_{L}$ for some scalar $s\left(w, w_{L}\right)$

$$
f(w)-f\left(w_{L}\right)=s\left(w, w_{L}\right)\left(w-w_{L}\right) .
$$

For $M>1, S_{L}$ consists of $M$ distinct one-parameter families, each originating from $w_{L}$ in the direction of $r_{k}\left(w_{L}\right) . w_{R}$ is on one of these one-parameter families; denote by $S_{L, R}$ the part of this one-parameter family which connects $w_{L}$ with $w_{R}$. We define the entropy condition for systems to be the same as the entropy condition (3.2c), where " $w$ between $w_{L}$ and $w_{R}$ " means $w \in S_{L, R}$ (see [17]). Similarly, we define the ACF $g$ for systems to be the same as (3.4), where $\alpha(w)$ is a scalar function, and " $w$ between $w_{L}$ and $w_{R}$ " again means that $w \in S_{L, R}$. The ACF $g$ (3.4) is now approximated by the vector 


$$
g_{j}^{n}=\alpha_{j}^{n}\left(w_{j+1}^{n}-w_{j-1}^{n}\right),
$$

where the nonnegative scalar $\alpha_{j}^{n}=\alpha_{j}\left(w^{n}\right)$ is defined by

$\alpha_{j}\left(w^{n}\right)=\max \left\{0, \min _{1 \leqslant m \leqslant M} \frac{\min \left[w_{m, j+1}^{n}-w_{m, j}^{n},\left(w_{m, j}^{n}-w_{m, j-1}^{n}\right) \operatorname{sgn}\left(w_{m, j+1}^{n}-w_{m, j}^{n}\right)\right]}{\left|w_{m, j+1}^{n}-w_{m, j}^{n}\right|+\left|w_{m, j}^{n}-w_{m, j-1}^{n}\right|}\right\}$.

Observe that for $M=1,(3.11)$ is identical to (3.7a) and that (3.7b) is satisfied for each of the components $g_{m . j}$ of the vector $g_{j}$ in (3.11). The artificial compression operator $C_{\Delta}$, for $M>1$, consists of applying the scalar one-sided scheme (3.6) to each of the components $w_{m}^{n}$ with $g_{m, j}^{n}=\alpha_{j}^{n}\left(w_{m, j+1}^{n}-w_{m, j-1}^{n}\right) ; \hat{\lambda}, 0 \leqslant \hat{\lambda} \leqslant 1$, is taken to be the same for all components and usually $\hat{\lambda}=1$.

In [9] and [10] we have presented examples of computations with the ACM $\hat{L}=C_{\Delta} L$, where $L$ is a monotone scheme and $C_{\Delta}$ is the artificial compressor described in this section. These computations exhibit oscillation-free transitions with excellent resolution of shocks and contact discontinuities. In fact since $\hat{L}=C_{\Delta}^{p} L$ with $p>1$ is also a possible operation, one can control the spread of discontinuities.

4. Self-Adjusting Hybrid Schemes. Let $L_{1}$ and $L_{R}$ be two conservative schemes such that $L_{1}$ is a nonoscillatory first order accurate scheme, $L_{R}$ is an $R$ th order accurate scheme with $R \geqslant 2$ :

$$
\begin{aligned}
& \left(L_{1} v\right)_{j}=v_{j}-\lambda\left(h_{j+1 / 2}^{1}-h_{j-1 / 2}^{1}\right), \\
& \left(L_{R} v\right)_{j}=v_{j}-\lambda\left(h_{j+1 / 2}^{R}-h_{j-1 / 2}^{R}\right) .
\end{aligned}
$$

As in [13] we consider self-adjusting hybrid schemes:

$$
\begin{aligned}
(L v)_{j} & =v_{j}-\lambda\left(h_{j+1 / 2}-h_{j-1 / 2}\right), \\
h_{j+1 / 2} & =\theta_{j+1 / 2} h_{j+1 / 2}^{1}+\left(1-\theta_{j+1 / 2}\right) h_{j+1 / 2}^{R} ;
\end{aligned}
$$

$\theta$ is a scalar quantity, $0 \leqslant \theta_{j+1 / 2} \leqslant 1 . \theta$, the automatic switch, is so constructed that $\theta \approx 1$, at discontinuities. There the behavior of the hybrid scheme is close to that of the nonoscillatory scheme $L_{1}$. Note that (4.2) is in conservation form.

By rewriting the hybrid scheme (4.2) as

$$
(L v)_{j}=\left(L_{R} v\right)_{j}+\lambda\left[\theta_{j+1 / 2}\left(h_{j+1 / 2}^{1}-h_{j+1 / 2}^{R}\right)-\theta_{j-1 / 2}\left(h_{j-1 / 2}^{1}-h_{j-1 / 2}^{R}\right)\right],
$$

we see that if $\theta$ is $O\left((\Delta x)^{p}\right)$ wherever the solution is smooth, then for $p \geqslant R-1$,

$$
\left(L v_{j}\right)=\left(L_{R} v\right)_{j}+O\left((\Delta x)^{R+1}\right)
$$

and the order of truncation error is preserved. We remark that the hybrid scheme (4.3a) can be regarded as the scheme $L_{R}$ modified by an adoptive artificial viscosity term. For example, when $L_{1}$ is the first order accurate Lax-Friedrichs scheme and $L_{2}$ is the second order accurate Lax-Wendroff scheme (LW), then (4.3a) takes the form of 


$$
\begin{aligned}
(L v)_{j}=\left(L_{2} v\right)_{j}+\frac{1}{2}\left[\theta_{j+1 / 2}\left(I-{ }^{2} A_{j+1 / 2}^{2}\right)\left(v_{j+1}-v_{j}\right)\right. \\
\\
\left.-\theta_{j-1 / 2}\left(I-{ }^{2} A_{j-1 / 2}^{2}\right)\left(v_{j}-v_{j-1}\right)\right] .
\end{aligned}
$$

Thus, hybridization adds a viscosity term to the LW scheme of the type

$$
\frac{(\Delta x)^{2}}{2}\left[\theta\left(I-\lambda^{2} A^{2}\right) v_{x}\right]_{x}
$$

(see [13]). Observe that if $\theta=O(\Delta x)$, then (4.4b) is of the same order as the truncation error in the LW scheme.

When we set $\theta$ to be a constant, $0 \leqslant \theta \leqslant 1$, then (4.2) becomes $L=\theta L_{1}+$ $(1-\theta) L_{R}$. It follows immediately from the inequality

$$
\|L\| \leqslant \theta\left\|L_{1}\right\|+(1-\theta)\left\|L_{R}\right\|
$$

that the hybrid scheme $L$ is stable if $L_{1}$ and $L_{R}$ are stable. It follows, therefore, that $L$ is stable under the stricter of the stability conditions of the two schemes $L_{1}$ and $L_{R}$. This statement remains valid in the presence of discontinuities, although the derivation, based upon the assumption of a locally constant $\theta$, becomes somewhat dubious. A much finer technique treating the discontinuity as a moving boundary is needed for a rigorous proof (see [6]).

In the following we shall deal primarily with Lax-Wendroff-type schemes, i.e. schemes that in the linear case $f(w)=A w, A=$ constant, are identical with the LW scheme $L_{2}$ :

$$
\begin{gathered}
\left(L_{2} v\right)_{j}=v_{j}-\frac{1}{2} \lambda A\left(v_{j+1}-v_{j-1}\right)+\frac{1}{2} \lambda^{2} A^{2}\left(v_{j+1}-2 v_{j}+v_{j-1}\right), \\
\lambda \max _{1 \leqslant k \leqslant M}\left|a_{k}\right| \leqslant 1 .
\end{gathered}
$$

The popular schemes of Richtmyer [23, pp. 330-338], MacCormack [19] and Thommen [25] are all schemes of this type. Each LW-type is hybridized with the corresponding first order accurate scheme

$$
\left(L_{1} v\right)_{j}=\left(L_{2} v\right)_{j}+\frac{1}{8}\left(v_{j+1}-2 v_{j}+v_{j-1}\right) .
$$

The linear stability condition of $L_{1}$ is

$$
\lambda \max _{1 \leqslant k \leqslant u}\left|a_{k}\right| \leqslant \frac{\sqrt{3}}{2} \approx 0.866 .
$$

The hybrid scheme $L$ composed of $L_{1}$ and $L_{2}$ is

$$
w_{j}=(L v)_{j}=\left(L_{2} v\right)_{j}+\frac{1}{8}\left[\theta_{j+1 / 2}\left(v_{j+1}-v_{j}\right)-\theta_{j-1 / 2}\left(v_{j}-v_{j-1}\right)\right] .
$$

$L$ is clearly stable under condition (4.7b) which is the stricter of the stability conditions of $L_{1}$ and $L_{2}$.

We now turn to analyze the linear scalar case. The LW scheme (4.6) is a linear second order accurate scheme and, therefore, is not monotonicity preserving. The first order accurate scheme (4.7) is monotonicity preserving, as is evident from the nonnega- 
tivity of the coefficients in $\left(L_{1} V\right)$, i.e.,

$$
\left(L_{1} v\right)_{j}=1 / 2(\mu+1 / 2)^{2} v_{j-1}+\left(3 / 4-\mu^{2}\right) v_{j}+1 / 2(\mu-1 / 2)^{2} v_{j+1} ;
$$

here $\mu=\lambda A$. To check whether the hybrid scheme (4.8) is monotonicity preserving, we follow the procedure of (2.7) and (2.8). Denote by $\Delta$ the differencing operator $\Delta_{j+1 / 2} v=v_{j+1}-v_{j}$. Let $v$ be any monotone mesh function and let $\delta$ be the sign of its variation ( $\delta=1$ if increasing, $\delta=-1$ if decreasing); thus $\delta \Delta_{j+1 / 2} v=\left|\Delta_{j+1 / 2} v\right|$. Subtracting (4.8) at $j=i$ from (4.8) at $j=i+1$, and then multiplying by $\delta$, we get after rearrangement that

$$
\begin{aligned}
\delta \Delta_{i+1 / 2} w= & 1 / 2\left[(\mu+1 / 2)^{2}-1 / 4\left(1-\theta_{i-1 / 2}\right)\right]\left|\Delta_{i-1 / 2} v\right| \\
& +\left[3 / 4-\mu^{2}+1 / 4\left(1-\theta_{i+1 / 2}\right)\right]\left|\Delta_{i+1 / 2} v\right| \\
& +1 / 2\left[(\mu-1 / 2)^{2}-1 / 4\left(1-\theta_{i+3 / 2}\right)\right]\left|\Delta_{i+3 / 2} v\right| .
\end{aligned}
$$

The hybrid scheme (4.8) is monotonicity preserving if and only if $\delta \Delta_{i-1 / 2} w \geqslant 0$. However, when $\mu=1 / 2,\left|\Delta_{i-1 / 2} v\right|=\left|\Delta_{i+1 / 2} v\right|=0,\left|\Delta_{i+3 / 2} v\right|>0$, we get from

$$
\delta \Delta_{i+1 / 2} w=-\frac{1}{8}\left(1-\theta_{i+3 / 2}\right)\left|\Delta_{i+3 / 2} v\right| \leqslant 0
$$

Similarly, when $\mu=-1 / 2,\left|\Delta_{i-1 / 2} v\right|>0,\left|\Delta_{i+1 / 2} v\right|=\left|\Delta_{i+3 / 2} v\right|=0$,

$$
\delta \Delta_{i+1 / 2} w=-\frac{1}{8}\left(1-\theta_{i-1 / 2}\right)\left|\Delta_{i-1 / 2} v\right| \leqslant 0 .
$$

We conclude from (4.10) that the property

$$
\theta_{j+1 / 2}=1 \quad \text { when }\left|\Delta_{j+1 / 2} v\right|>0, \quad\left|\Delta_{j-1 / 2} v\right|\left|\Delta_{j+3 / 2} v\right|=0
$$

is a necessary condition for the hybrid scheme (4.8) to be monotonicity preserving. Observe that condition (4.11) corresponds to a case where the solution has a sharp corner, which typically occurs at the end points of a jump discontinuity.

Next we describe an automatic switch which satisfies condition (4.11). Let $\sigma(w)$ be a function of $w$ which is discontinuous at shocks and at contact discontinuities; denote by $\sigma_{j}=\sigma\left(v_{j}\right), \Delta_{j+1 / 2} \sigma=\sigma_{j+1}-\sigma_{j}$, and define

$$
\hat{\theta}_{j}= \begin{cases}\left|\frac{\left|\Delta_{j+1 / 2} \sigma\right|-\left|\Delta_{j-1 / 2} \sigma\right|}{\left|\Delta_{j+1 / 2} \sigma\right|+\left|\Delta_{j-1 / 2} \sigma\right|}\right|^{p} & \text { for }\left|\Delta_{j+1 / 2} \sigma\right|+\left|\Delta_{j-1 / 2} \sigma\right|>\epsilon, \\ 0 & \text { for }\left|\Delta_{j+1 / 2} \sigma\right|+\left|\Delta_{j-1 / 2} \sigma\right| \leqslant \epsilon .\end{cases}
$$

Here $\epsilon>0$ is some suitably chosen measure of insignificant variation in $\sigma(w)$. It is easy to see that $\hat{\theta}_{j}=O\left((\Delta x)^{p}\right)$ wherever the solution is smooth; $0 \leqslant \hat{\theta}_{j} \leqslant 1$, and $\hat{\theta}_{j}=1$ only when either $\Delta_{j+1 / 2} \sigma=0$ and $\left|\Delta_{j-1 / 2} \sigma\right|>\epsilon$ or when $\left|\Delta_{j+1 / 2} \sigma\right|>\epsilon$ and $\Delta_{j-1 / 2} \sigma=0$. We define the automatic switch by

$$
\theta_{j+1 / 2}=\max \left(\hat{\theta}_{j}, \hat{\theta}_{j+1}\right) \text {. }
$$

It follows immediately that $0 \leqslant \theta_{j+1 / 2} \leqslant 1$ and that $\theta_{j+1 / 2}$ satisfies the corner condition (4.11). 
Analysis of a general $2 k+1$ point hybrid scheme, based on the same reasoning as (2.7), (2.8), (4.10) and (4.11), suggests defining $\theta_{j+1 / 2}$ by

$$
\theta_{j+1 / 2}=\max \left(\hat{\theta}_{j-k+1}, \ldots, \hat{\theta}_{j+k}\right) \text {. }
$$

We would like to point out that (4.12) is a very sensible way to define an automatic switch. There are two ways to detect a discontinuity on a fixed mesh:

1. Look for a large variation of the solution. This is a tricky business which usually requires a certain familiarity with the solution; a switch so constructed will usually have its maximum at the center of the transition (see [13]).

2. Look for abrupt changes in the variation of the solution; such abrupt changes occur at the end points of the transition.

The switch (4.12) follows the second recipe. $\hat{\theta}_{i}$ in (4.12a) is a sensor of abrupt changes in the variation of the solution (this was already observed by Van Leer in [26]); and the switch $\theta_{i+1 / 2}$ in (4.12b) is $\approx 1$ at the end points of a transition of a discontinuity. Since the presence of a corner in the solution drives nonmonotone schemes to generate oscillations, avoidance of oscillations requires the use of a monotonicity preserving scheme at corners; a switch which is $\approx 1$ at corners does just that.

THEOREM. Let $\theta_{j+1 / 2}$ be the automatic switch (4.12) defined with $\sigma(w)=w$ and $p=1$. Then the hybrid scheme (4.8), where $L_{2}$ is the Lax-Wendroff scheme (4.6), is monotonicity preserving for at least $|\mu| \leqslant \sqrt{2} / 2$.

A proof is given in the appendix.

The switch (4.12) detects abrupt changes in the variation of $\sigma(w)$, independent of their size. When $\sigma(w)$ is constant for the exact solution, its values for the numerical solution might fluctuate and, in so doing, trigger the switch. Such an occurrence can be prevented by choosing an appropriate tolerance $\epsilon$ in (4.12a).

We now turn to a discussion of hybridization for the case of systems of equations. It is possible to extend the definition of the automatic switch (4.12) by

1. Taking $\sigma(w)$ to be a vector function of $w$ and then replacing the absolute values in $(4.12 \mathrm{a})$ by norms, or

2. Taking $\sigma(w)$ to be a scalar function of the vector $w$. $\sigma$ is to exhibit the following properties: $\sigma$ will have a jump discontinuity when $w$ is a shock or a contact discontinuity; otherwise it is smooth. Equation (4.12) then need not be modified.

The second possibility, because it seems computationally more attractive is used in the following computations. As in the scalar case, $\sigma(w)$ forms a corner at the end points of an abrupt transition; consequently, $\theta \approx 1$ there, so that at such a point the hybrid scheme (4.8) is almost the same as the nonoscillatory $L_{1}$. Thus, we expect the hybrid scheme (4.8) for systems to produce transitions which are practically oscillationfree. Figures 1-3 show numerical solutions of (4.8) to the following Riemann problem for the Eulerian equations of a polytropic gas:

$$
w_{t}+f(w)_{x}=0
$$

$$
w=\left(\begin{array}{c}
\rho \\
m \\
E
\end{array}\right), \quad f(w)=u w+\left(\begin{array}{c}
0 \\
P \\
P u
\end{array}\right), \quad P=(\gamma-1)\left(E-1 / 2 \rho u^{2}\right)
$$




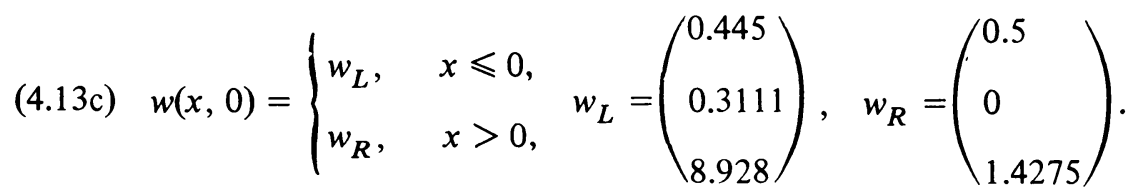

Here $\rho, u, P$ and $E$ are the density, velocity, pressure and total energy, respectively; $m=\rho u$ is the momentum, $\gamma=1.4$. The exact solution of this Riemann problem, which consists of a shock propagating to the right followed by a contact discontinuity, and a left propagating rarefaction, is shown in the figures (continuous line). $L_{2}$ in (4.8) is the MacCormack scheme [19]:

$$
\begin{aligned}
& \bar{v}_{j}^{n+1}=v_{j}^{n}-\lambda\left[f\left(v_{j+1}^{n}\right)-f\left(v_{j}^{n}\right)\right], \\
& v_{j}^{n+1}= \frac{1}{2}\left(v_{j}^{n}+\bar{v}_{j}^{n+1}\right)-\frac{\lambda}{2}\left[f\left(\bar{v}_{j}^{n+1}\right)-f\left(\bar{v}_{j-1}^{n+1}\right)\right] \\
&+\frac{1}{8}\left[\theta_{j+1 / 2}^{n}\left(v_{j+1}^{n}-v_{j}^{n}\right)-\theta_{j-1 / 2}^{n}\left(v_{j}^{n}-v_{j-1}^{n}\right)\right] .
\end{aligned}
$$

Figures 1-3 show the solution of (4.14) after 200 time steps under the condition

$$
\frac{\Delta t^{(n)}}{\Delta x} \max \left(\left|u^{n}\right|+c^{n}\right)=0.95 \beta
$$

where $c=(\gamma P / \rho)^{1 / 2}$ is the sound speed and $\Delta x=0.1$.

Figure 1 shows the second order accurate MacCormack scheme, i.e. $\theta \equiv 0$ in (4.14); $\beta$ in (4.15) is 1 . Observe the large overshoots at the shock and the large undershoots at the contact discontinuity. The resolution of the shock is much better than that of the contact discontinuity; the latter is smeared at the rate $n^{1 / 3}$. Note that although the rarefaction wave is accurately computed oscillations are generated at its right end point.

Figure 2 shows the solution of the first order accurate scheme (4.7), i.e., $\theta \equiv 1$ in (4.14); $\beta$ in (4.15) is $\sqrt{3} / 2$. Observe that the solution is oscillation-free. The transition of the shock occupies 5-6 cells; the transition of the contact discontinuity, which is being smeared at the rate $n^{1 / 2}$, occupies about 25 cells $(n=200)$. The constant state between the contact discontinuity and the shock is not fully realized. Note the excessive rounding of the corners at the end points of the rarefaction wave.

Figure 3 shows the solution of the hybrid scheme (4.14) with $\theta_{j+1 / 2}^{n}$ defined by (4.12) with $\sigma(w)=\rho$ and $p=1 ; \beta$ in (4.15) is $\sqrt{3} / 2$. Observe that the solution is oscillation-free and that the resolution of the discontinuities is better than that of the first order accurate scheme in Figure 2. The transition of the shock occupies 3-4 cells; the transition of the contact discontinuity occupies 14-15 cells. Note that the rarefaction wave is also resolved more accurately than the one in Figure 2; the corners at the end points of the rarefaction wave are only slightly rounded.

The restriction on the size of the time step $(\beta$ in (4.15)) in the first order scheme (4.7) and in the hybrid scheme $(4.8)(\beta=\sqrt{3} / 2)$ is $13.4 \%$ more severe than that in the MacCormack scheme $(\beta=1)$. It is interesting to note that the total time after 200 time steps, is $T=3.5$ for the two computations shown in Figures 2 and 3 and only $T=2.9$ for the calculation shown in Figure 1 . 

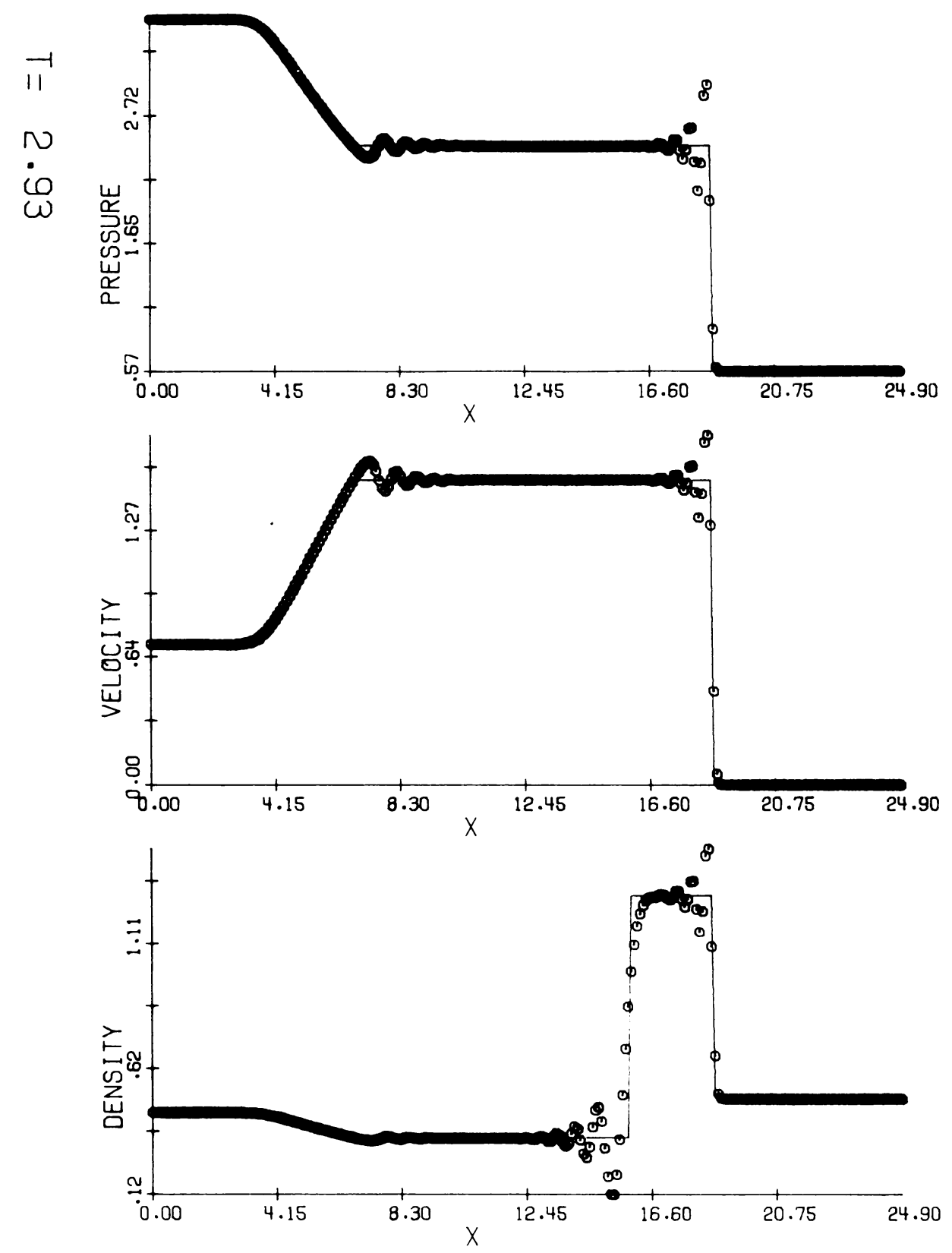

FIGURE 1. MacCormack scheme (4.14)

5. Self-Adjusting Hybrid ACM. In Section 4 we have shown how to hybridize an $R$ th order accurate scheme $L_{R}$ with a nonoscillatory first order accurate scheme $L_{1}$ so that the hybrid scheme $L$ is almost $L_{1}$ at discontinuities and yet $L-L_{R}=$ $O\left((\Delta x)^{R+1}\right)$ wherever the solution is smooth. Discontinuities computed with such a hybrid scheme are oscillation-free, although smeared. In Section 3 we have shown how to correct the smearing of discontinuities in first order accurate nonoscillatory schemes by ACM. The ACM described there is based on the assumption that in the numerical solution, a discontinuity is approximated by a continuous but rapid transition, very similar to nonoscillatory viscous profiles. Consequently, the ACM is inherently limited 

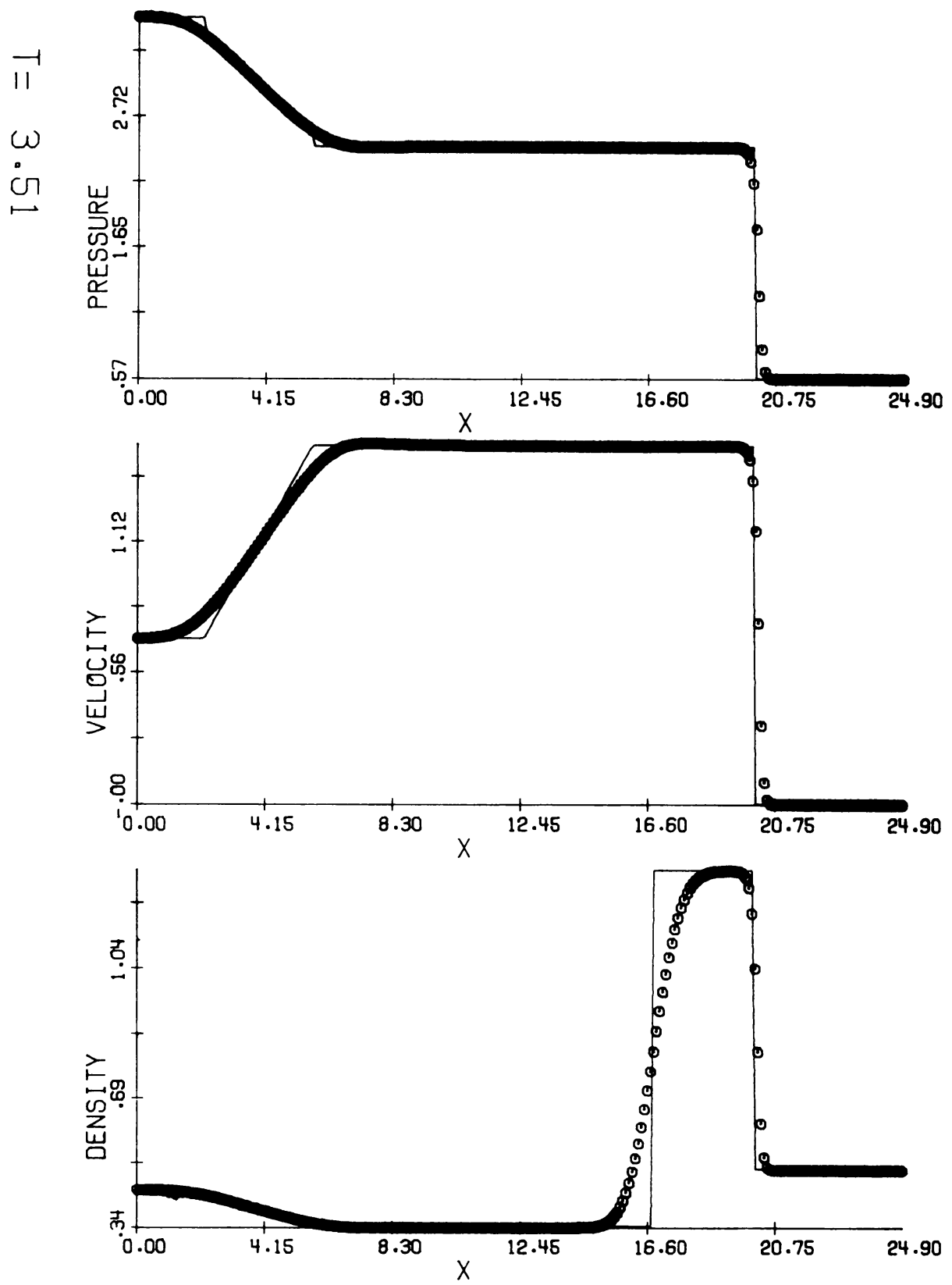

FIGURE 2. The first order scheme (4.7)

to nonoscillatory first order accurate schemes; attempts to apply ACM to high order accurate schemes result in an increase of overshoot and in some cases convergence to a nonphysical solution (see [11]) occurs. In this section we show how the combination of high order schemes, hybridization and ACM leads to the "ultimate scheme".

We consider a corrective type $\operatorname{ACM} \hat{L}=C_{\Delta} L ; L$ is now a nonoscillatory hybrid scheme (4.2) for the solution of the original problem (2.1), e.g. scheme (4.14). Since $L \approx L_{1}$ at discontinuities, the use of the first order accurate ACM of Section 3 is meaningful; the application of $C_{\Delta}$ to $L v$ corrects the smearing of a discontinuity by $L$ 


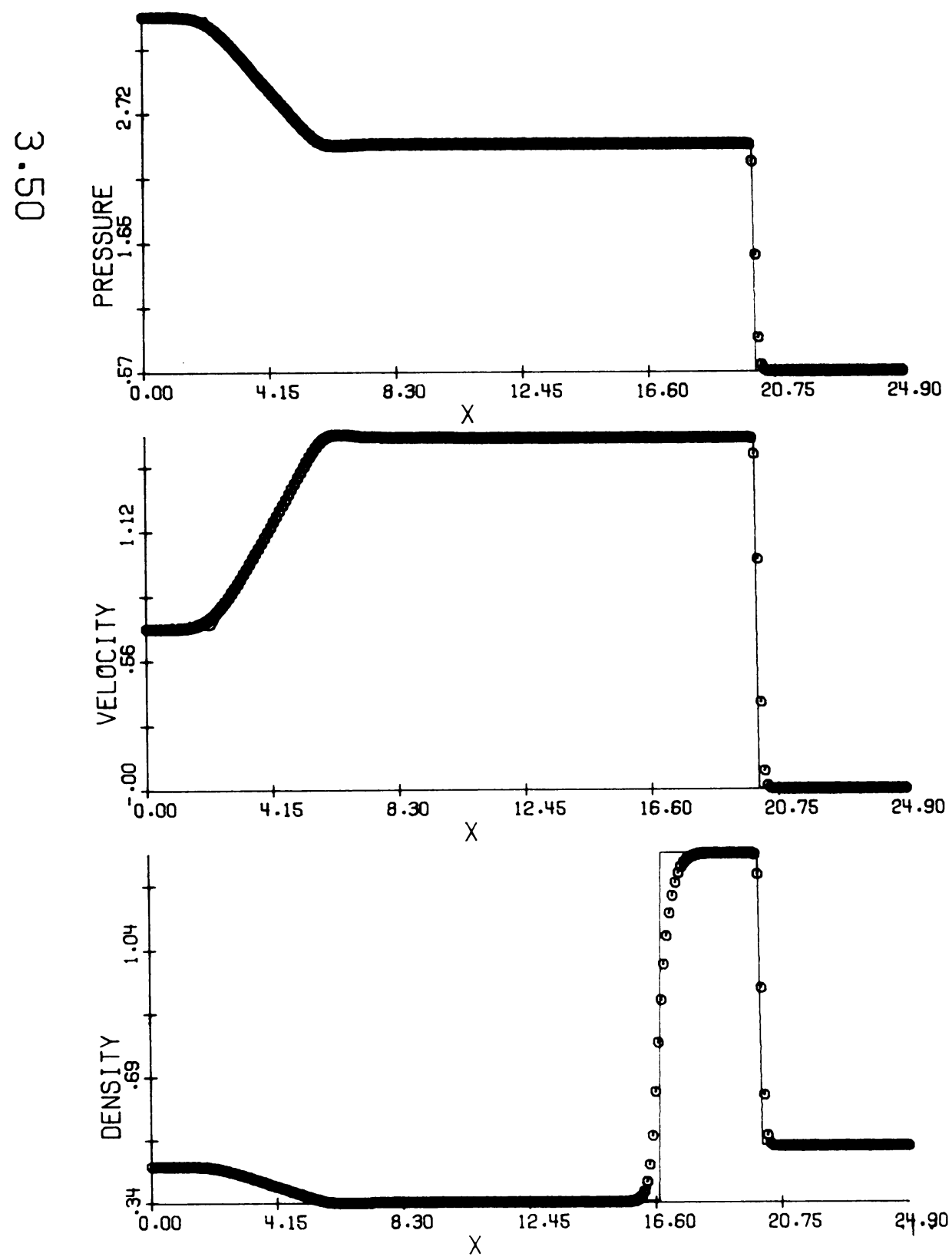

FIGURE 3. The hybrid scheme (4.14)

and brings the discontinuity closer to its maximal resolution (3.8b). This, due to the corner property (4.11) of the automatic switch (4.12), makes the change of $L$ into $L_{1}$ even more complete. Therefore, we expect $\hat{L}=C_{\Delta} L$ to produce the same oscillationfree sharp transitions of the discontinuities as in [10]. To preserve the high order of the truncation error of $L_{R}$ in regions of smoothness of the solution, we switch off the first order accurate ACM in these regions and switch on the infinitely accurate identity operator $I v=v$. This leads to the following hybrid artificial compressor 

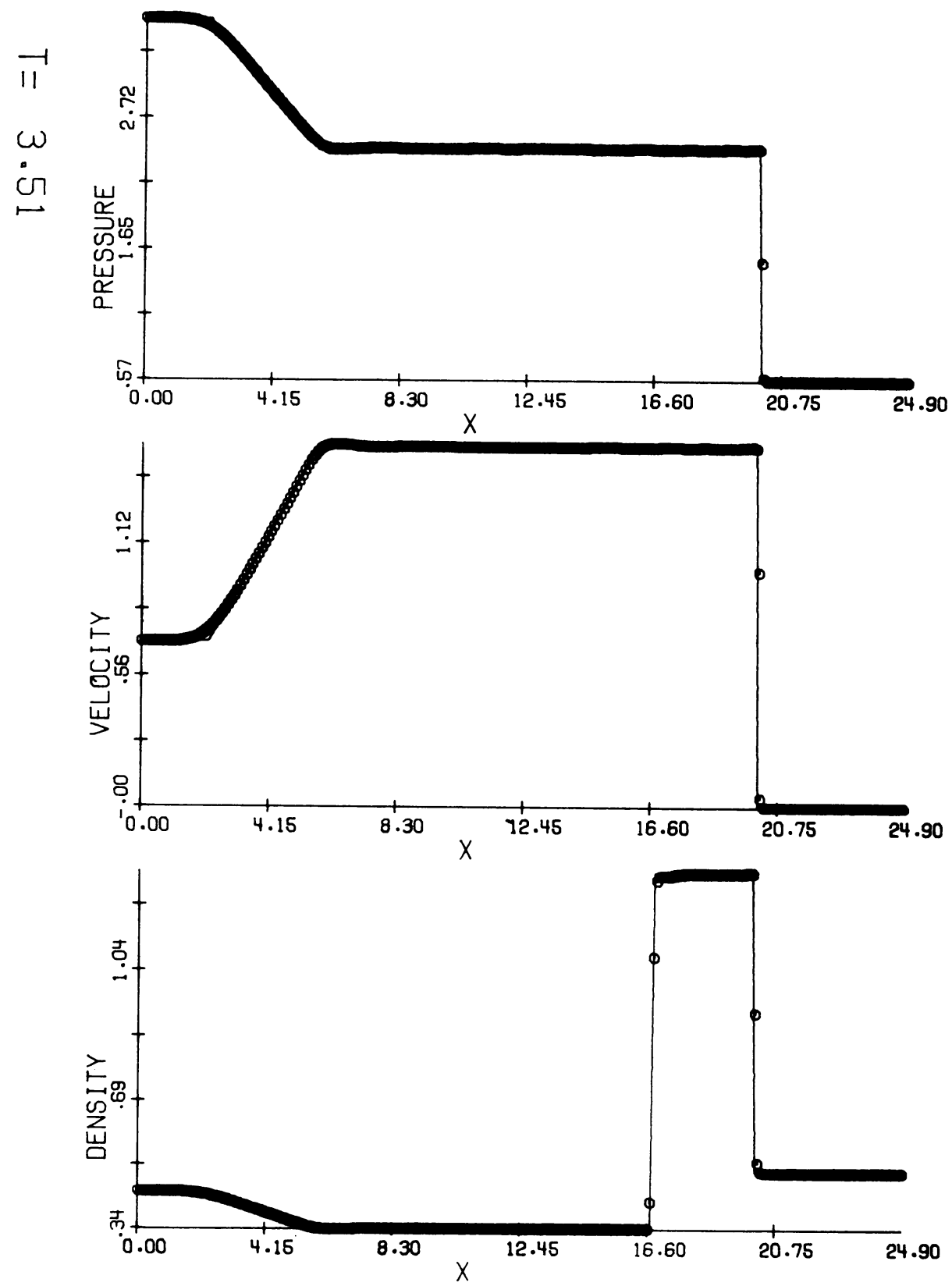

FIgURE 4. The hybrid $A C M \hat{L}=C_{\Delta} L,(4.14)$ and (5.1)

$$
\left(C_{\Delta} v\right)_{j}=v_{j}-(\hat{\lambda} / 2)\left(\theta_{j+1 / 2} G_{j+1 / 2}-\theta_{j-1 / 2} G_{j-1 / 2}\right) ;
$$

$G_{m, j+1 / 2}$, the $m$ th component of the vector $G_{j+1 / 2}$, is given by

(5.1b) $G_{m, j+1 / 2}=g_{m, j}+g_{m, j+1}-\left|g_{m, j+1}-g_{m, j}\right| \operatorname{sgn}\left(v_{m, j+1}-v_{m, j}\right)$,

where

$$
g_{m, j}=\alpha_{j}(v)\left(v_{m, j+1}-v_{m, j-1}\right)
$$



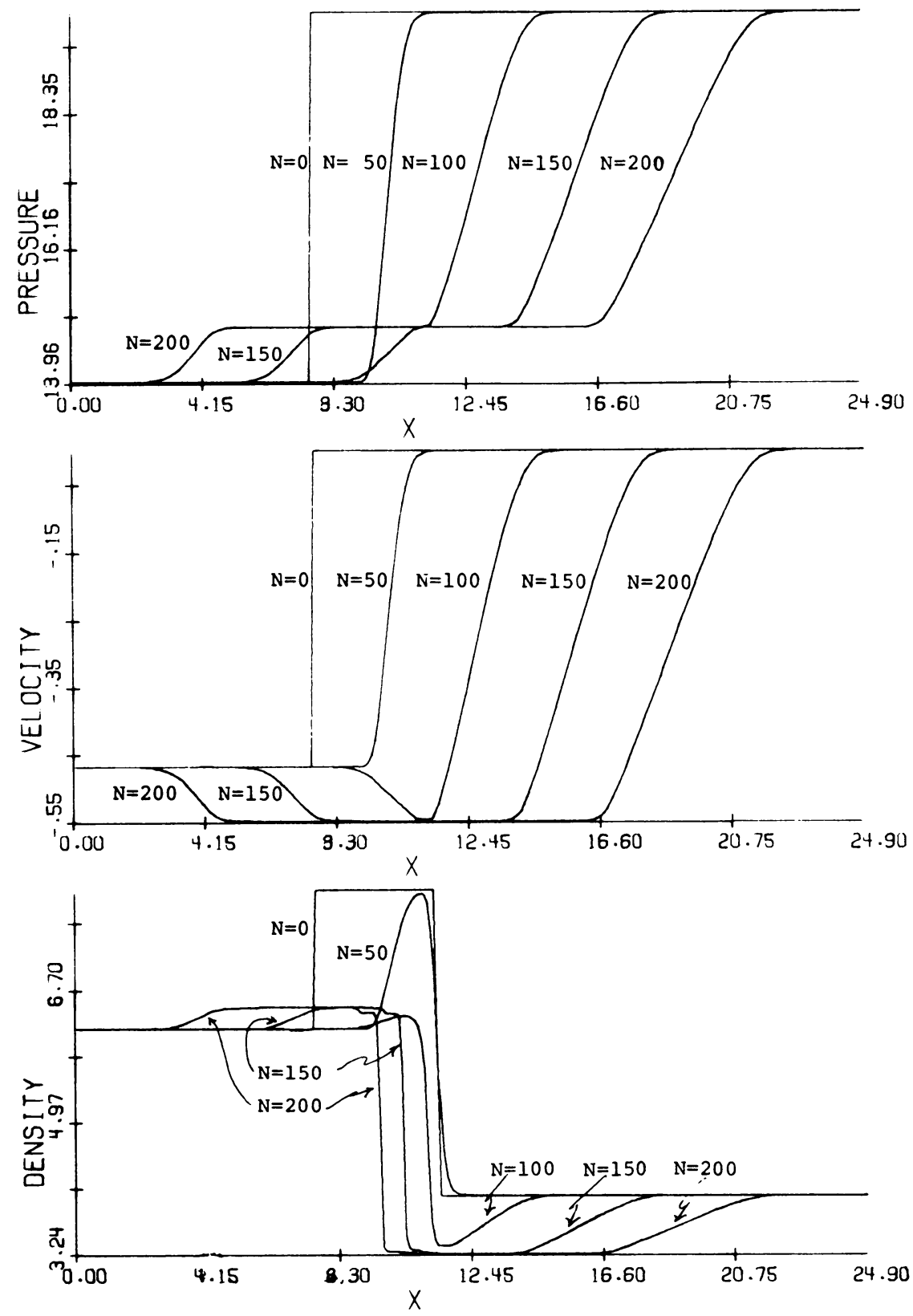

FIGURE 5. Time evolution of an interaction between a rarefaction wave and a contact discontinuity

and $\alpha_{j}(v)$ is defined by (3.11b). $\theta_{j+1 / 2}$ is the automatic switch (4.12a)-(4.12c) with $p \geqslant R-1$. The hybrid artificial compressor (5.1) is stable under $\hat{\lambda} \leqslant 1$ (we usually take $\hat{\lambda}=1$ ) and is completely independent of the choice of $L$, as long as $L$ is a nonoscillatory scheme. The automatic switch in the artificial compressor (5.1) need not be the same as that given by (4.2).

The only function of the hybrid ACM (5.1) is to improve resolution of the computed solution in the neighborhood of admissible discontinuities; in regions devoid of 

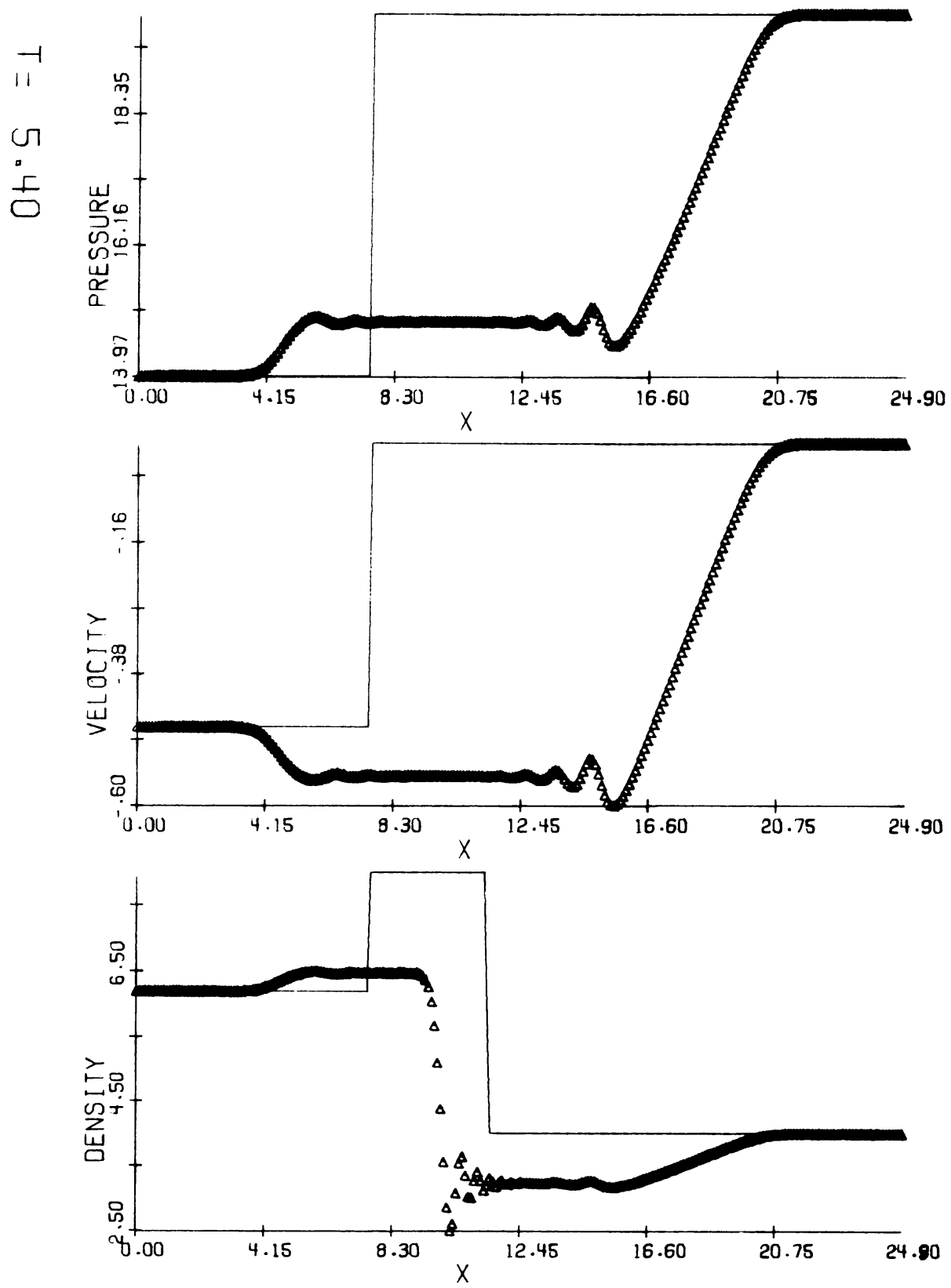

FIGURE 6. Richtmyer scheme (5.3)

the discontinuities, the artificial compression does not improve the accuracy of the computation. In regions where $\left|v_{x x}\right|$ is bounded, the operator (5.1) preserves the order of truncation error in $L_{R}$; thus the formal order of accuracy is not affected. However, in regions where $v_{x x}$ is unbounded, such as at inadmissible discontinuities or at end points of a centered rarefaction wave, the artificial compression should not be applied because it might interfere with the proper evolution of the numerical solution. To restrict the region where $C_{\Delta}$ contributes to the solution to the immediate vicinity of admissible discontinuities, we need, in (4.12), a function $\sigma(w)$ that exhibits a jump 

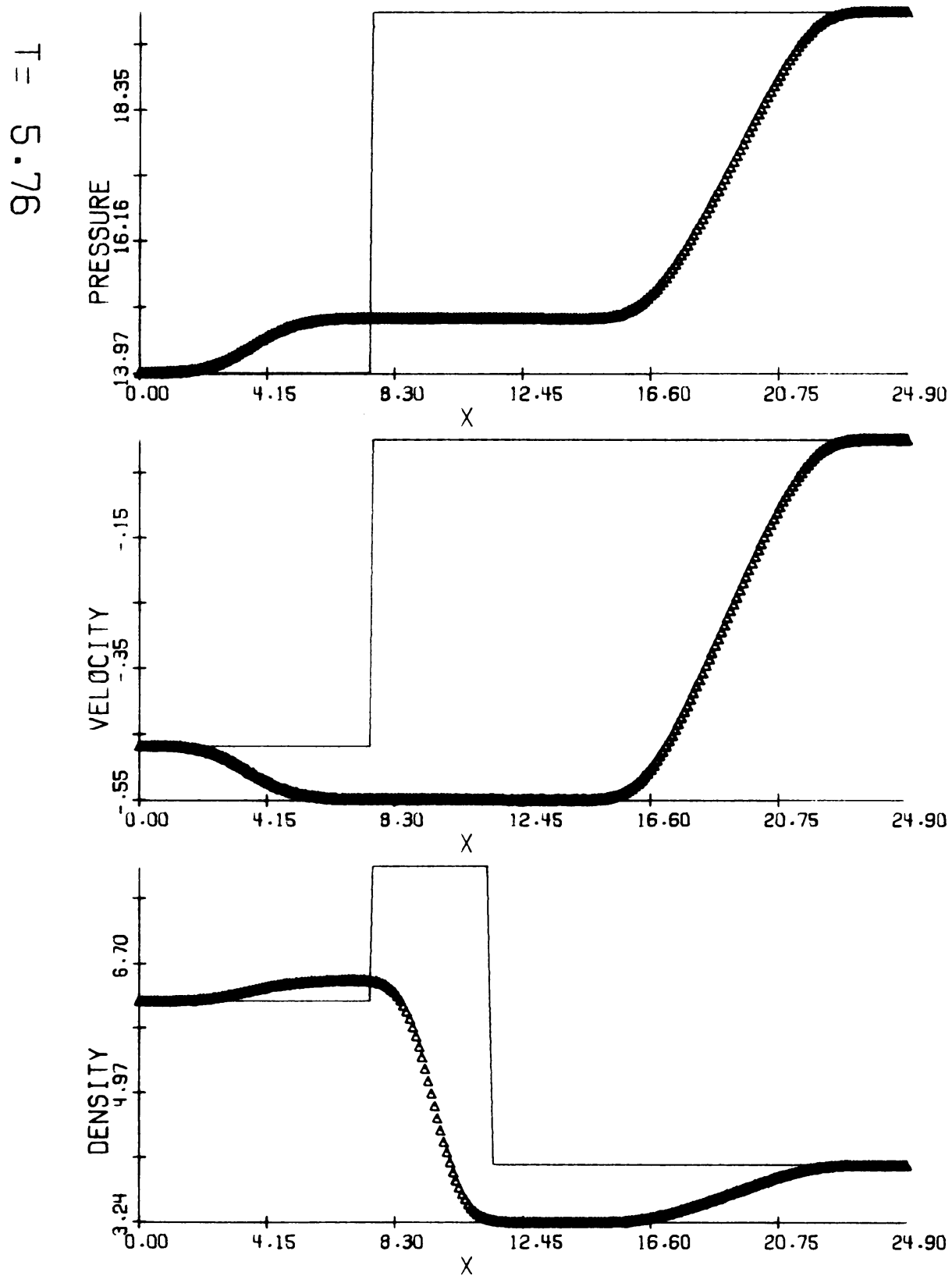

FIGURE 7. The first order scheme (5.4) with $\theta \equiv 1$

discontinuity at shocks and contact discontinuities with little variation otherwise. A natural thermodynamic function is readily available: entropy. The automatic switch (4.12) with $\sigma(w)$ identified with the entropy will be referred to as the entropy switch. As was mentioned in Section 4, (4.12) detects abrupt changes in the variation of $\sigma(w)$, independent of their size. Although entropy in the exact solution is constant except for jump discontinuities at shocks and contact discontinuities, its value for the numerical solution might fluctuate and as such trigger the switch. To prevent such an occurrence, one should choose in (4.12a) an appropriate tolerance $\epsilon$, which is larger than the 

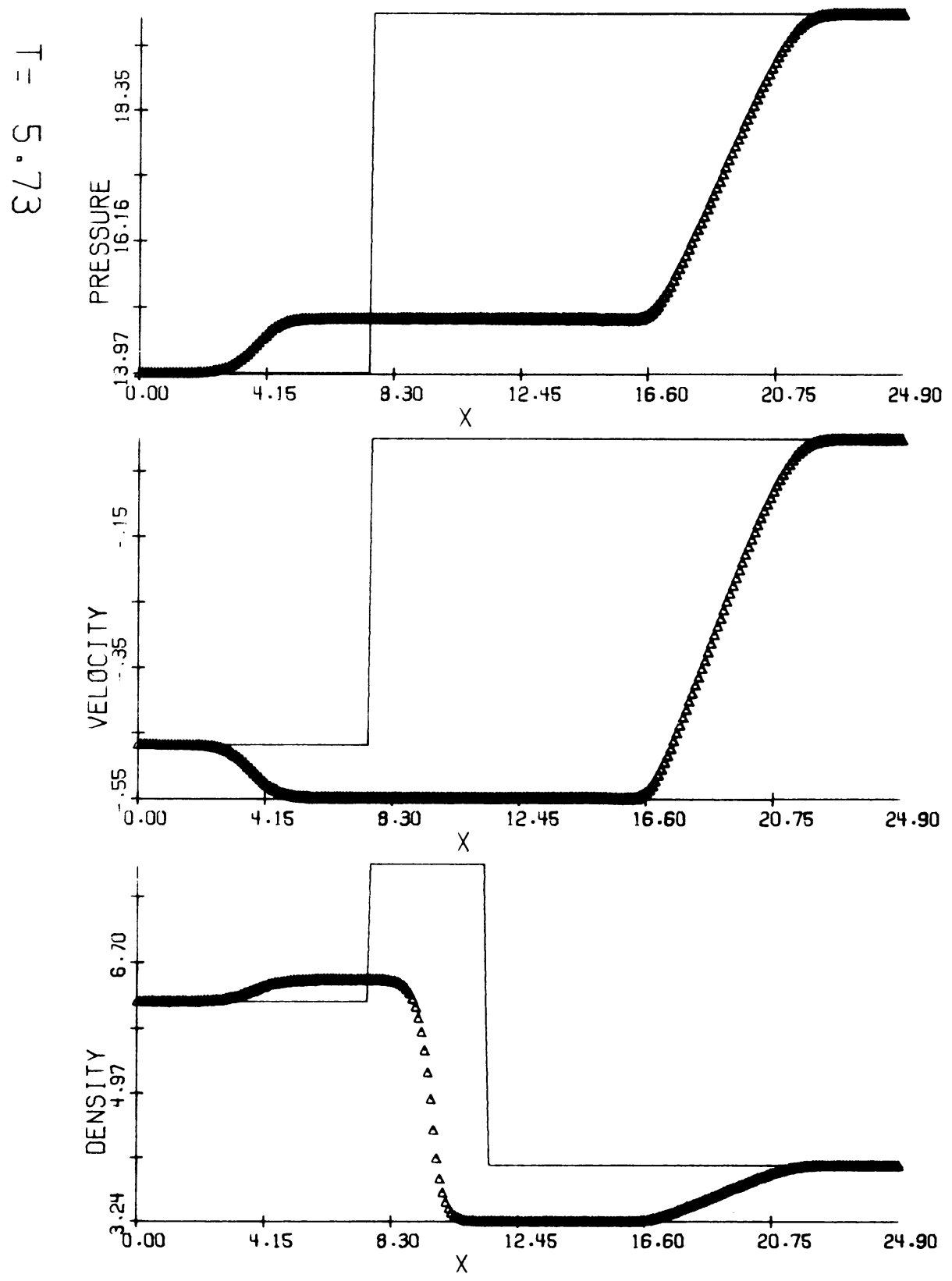

FIGURE 8. The hybrid scheme (5.4)

maximal numerical fluctuation in the entropy.

The entropy switch is somewhat expensive to compute. Following Rosenbluth's idea [23, p. 310], we can use one of the already computed variables, such as $\sigma(w)=\rho$, to construct the automatic switch (4.12), and set it to zero in regions of expansion. In the case of Eulerian gas equations we set $\hat{\theta}_{j}$ in $(4.12 a)$ to zero when:*

${ }^{*}$ I thank G. Kleinstein for suggesting this test. 

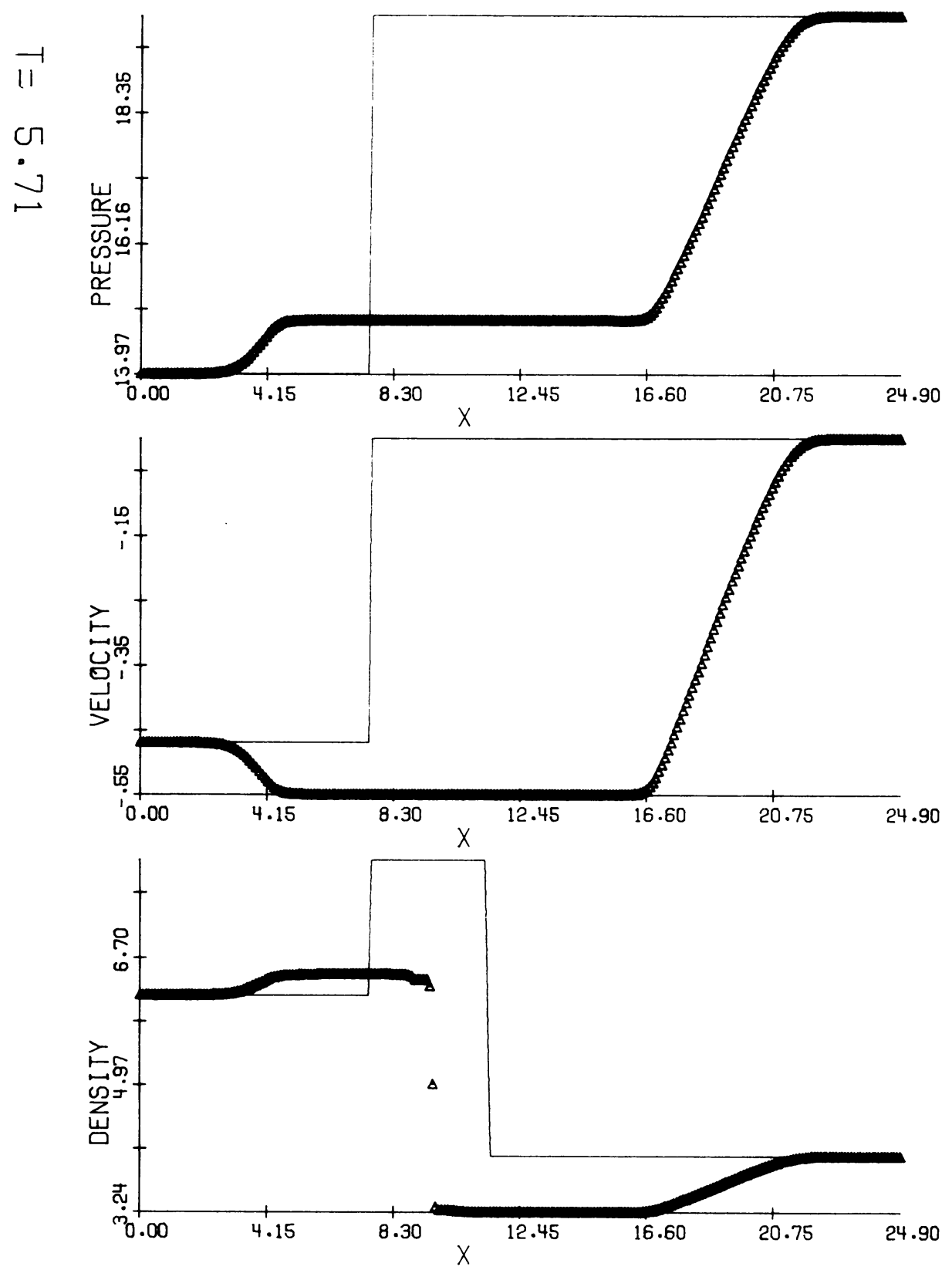

Figure 9. The hybrid $A C M \hat{L}=C_{\Delta} L$, (5.4) and (5.1)

$$
\frac{(\rho u)_{j+1}-(\rho u)_{j-1}}{\rho_{j+1}-\rho_{j-1}} \operatorname{sgn}\left(u_{j}\right)<0 .
$$

We recommend using the test (5.2) even while using the entropy switch; this is a precaution against an inappropriate choice of the tolerance $\epsilon$. We remark that part of the extra computing time spent on the test (5.2) (or on calculating entropy) is regained, since we set $G_{j+1 / 2}=0$ wherever $\theta_{j+1 / 2}=0$; thus $G_{j+1 / 2}$ is actually evaluated only at a few points. 
Figure 4 shows the solution of the Riemann problem (4.13) after 200 time steps under condition (4.15) with $\beta=\sqrt{3} / 2$ of the scheme $\hat{L}=C_{\Delta} L$, where $L$ is the selfadjusting MacCormack scheme (4.14) and $C_{\Delta}$ is the artificial compressor (5.1). The automatic switch in both $L$ and $C_{\Delta}$ is (4.12) with $\sigma(w)=\rho$ and $p=1$, with the provision that $\hat{\theta}_{j}$ in (4.12a) for the artificial compression is set to zero when condition (5.2) is satisfied. The discontinuities are practically oscillation-free and their resolution is almost maximal; if better resolution is needed, one can apply $C_{\Delta}$ one more time so that in effect $\hat{L}=C_{\Delta}^{2} L$. Observe that the constant state between the contact discontinuity and the shock is fully realized (the error is in the 4th significant figure); the rarefaction wave is the same as for the hybrid scheme in Figure 3.

Figures 5-9 show numerical solutions of an interaction between a rarefaction wave and a stationary contact discontinuity (see [4, pp. 197-199]). The centered rarefaction wave starts from a jump discontinuity situated to the left of the contact discontinuity ( $N=0$ in Figure 5 and the continuous line in Figures 6-9), and propagates toward it. As the rarefaction wave hits the contact discontinuity $(N=50$ in Figure 5), it is partially transmitted and partially reflected. As a result of the interaction, the contact discontinuity propagates to the left with a constant velocity $(N=$ 100,150 and 200 in Figure 5).

Figure 6 shows the solution after 200 time steps under condition (4.15) with $\beta=1$ of the Richtmyer scheme $v^{n+1}=L_{2} v^{n}$ :

$$
\begin{aligned}
v_{j+1 / 2}^{n+1 / 2} & =\frac{1}{2}\left(v_{j}^{n}+v_{j+1}^{n}\right)-\frac{1}{2} \lambda\left[f\left(v_{j+1}^{n}\right)-f\left(v_{j}^{n}\right)\right], \\
v_{j}^{n+1} & =v_{j}^{n}-\lambda\left[f\left(v_{j+1 / 2}^{n+1 / 2}\right)-f\left(v_{j-1 / 2}^{n+1 / 2}\right)\right] .
\end{aligned}
$$

Figures 7 and 8 show the solution after 200 time steps; the solution was computed subject to stability condition (4.15) with $\beta=\sqrt{3} / 2$ and the difference operator

$$
v_{j}^{n+1}=\left(L v^{n}\right)_{j}=\left(L_{2} v^{n}\right)_{j}+\frac{1}{8}\left[\theta_{j+1 / 2}^{n}\left(v_{j+1}^{n}-v_{j}^{n}\right)-\theta_{j-1 / 2}^{n}\left(v_{j}^{n}-v_{j-1}^{n}\right)\right]
$$

with $L_{2}$ designating the system (5.3). Figure 7 shows the solution of the first order scheme (5.4) with $\theta \equiv 1$. Figure 8 shows the solution of the hybrid scheme (5.4), where the automatic switch $\theta_{j+1 / 2}$ is defined by (4.12) with $\sigma(w)=\rho$ and $p=1$.

Figure 9 shows the solution obtained from the hybrid ACM $\hat{L}=C_{\Delta} L$ after 200 time steps. Here $L$ is the same as in Figure 8 and $C_{\Delta}$ is the artificial compressor (5.1). The automatic switch $\theta$ in the artificial compressor is (4.12) with $\sigma(w)=$ entropy, with the provision that $\hat{\theta}_{j}$ in $(4.12 \mathrm{a})$ is set to zero when condition (5.2) is satisfied; $\epsilon$ in (4.12a) is taken to be $\epsilon^{n}=0.01 \max _{j}\left|\Delta_{j+1 / 2} \sigma^{n}\right|$. The result shown in Figure 5 was computed exactly the same way as the result shown in Figure 9. Observe that there is a slight "step" in the constant state on the left of the contact discontinuity. We consider this "step phenomenon" to be tolerable; if desired, it can be eliminated by an operation described in [10] which increases the convexity of the ACF (3.11). Note that maximal resolution of the contact discontinuity is achieved and that there is only a slight rounding of the corners at the end points of the rarefaction waves. 
6. To Hybridize or Not to Hybridize: That is the Question. There are many important questions in shock computations which are still open. As a result, many ideas are still controversial and new approaches require justification; we devote this section to justification. We argue, that the idea of the self-adjusting hybrid ACM does make sense. The main points in the argument are:

(i) The change to a nonoscillatory first order accurate scheme at discontinuities is physically and mathematically desirable.

(ii) This change does not damage the accuracy at discontinuities and in fact improves it.

(iii) Even in the presence of discontinuities there is a gain in using high order accurate schemes wherever the solution is smooth. We elaborate.

(i) Shock waves in the physical world as well as in mathematical thought are usually conceived as a result of some limiting process. In the physical world shocks are discontinuities which are conceptualized as a boundary layer phenomenon with vanishing small boundary layer thickness. Mathematical theory, because of nonuniqueness of discontinuous solutions of the inviscid problem (2.1), relies upon a limiting process to determine which of the solutions is a physical one, i.e., it admits only those discontinuities which can be realized as the limit $\epsilon \rightarrow 0$ of the viscous equation

$$
w_{t}+f(w)_{x}=\epsilon w_{x x} .
$$

Numerical schemes behave very much like the solution operator of the corresponding modified equations; in particular first order monotone schemes behave like the viscous equation (2.5). The limit $\Delta t \rightarrow 0$ in (2.5) mimics the limit $\epsilon \rightarrow 0$ in (6.1), thus providing monotone schemes with a built-in selection mechanism to determine the physically relevant solutions. Oscillations at discontinuities in solutions of nonmonotone schemes introduce nonphysical states that might trigger the selection of an inadmissible solution. Solutions to problems where the flux $f(w)$ in $(2.1)$ is nonconvex, are especially susceptible to such occurrences. Therefore, switching to a nonoscillatory first order accurate scheme at discontinuities is a natural thing to do.

(ii) Does the use of high order accurate schemes to compute discontinuities improve the local accuracy of the computation? To answer this question we study a computation of an admissible discontinuity $\left(w_{L}, w_{R}, S\right)$ with $S \neq 0$. Let $w^{n}$ be the solution of any conservative finite-difference scheme after $n$ time steps. Denote by $z(x, t)$ the exact solution

$$
z(x, t)= \begin{cases}w_{L}, & x \leqslant S t \\ w_{R}, & x>S t .\end{cases}
$$

The initial data $w_{j}^{0}=z(j \Delta x, 0)$

$$
w_{j}^{0}= \begin{cases}w_{L}, & j \leqslant 0, \\ w_{R}, & j>0,\end{cases}
$$

remains unchanged under refinement of the mesh. The finite-difference operation itself depends only on $\lambda=\Delta t / \Delta x$. Thus, the standard refinement process $\Delta x \rightarrow 0$ with $\lambda$ 
fixed is the same as $n \rightarrow \infty$. The first order accurate ACM approaches, as $n \rightarrow \infty$, a steady progressing profile with an almost optimal resolution ( $3.8 \mathrm{~b})$ for both shocks and contact discontinuities (see [9] and [10]). These steady progressing profiles also have the least $l_{2}$-error, $e(T)$ for $T=n \Delta t$ :

$$
e(T)=\left[\Delta x \sum_{j=-\infty}^{\infty}\left|w_{j}^{n}-z(j \Delta x, n \Delta t)\right|^{2}\right]^{1 / 2} .
$$

As $\Delta x$ decreases (same as $n$ increases), the sum in (6.3) tends to a constant; thus the $l_{2}$-error in the ACM is $e(T)=O\left((\Delta x)^{1 / 2}\right)$ for both shock and contact discontinuity. Standard $R$ th order accurate schemes smear a contact discontinuity as well as a scalar linear discontinuity at the rate of $n^{1 /(R+1)}$; consequently, the $l_{2}$-error (6.3) becomes $e(T)=O\left((\Delta x)^{0.5 R /(R+1)}\right.$ ) (see [22] and [8]). Although $1 / 2 R /(R+1)$ is an increasing function of $R$, it is smaller than the optimal $O\left((\Delta x)^{1 / 2}\right)$ of the ACM for all finite $R$.

(iii) It follows from the above analysis that in the presence of discontinuities there are regions in the numerical solution in which the local $l_{2}$-error is at best $O\left((\Delta x)^{1 / 2}\right)$. Does this error spread into regions where the solution is smooth? If so, does the error produced at the discontinuity dominate the error produced by the higher order schemes making such schemes ineffective? At present there is a clear cut answer only in the case of a shock in the solution of a scalar equation. In this case the characteristic field which is convergent towards the line $d x / d t=S$ at the locality of the shock, acts to confine the $O\left((\Delta x)^{1 / 2}\right)$ error to this region. The $O\left((\Delta x)^{R}\right)$ error of an $R$ th order accurate scheme is, therefore, preserved for points sufficiently far away from the shock. We cannot use the same argument to show such a containment of error mechanism in the case of systems, as out of the $2 M$ characteristics on both sides of the shock (see [17]) only $M+1$ characteristics converge towards $d x / d t=S$, while $\boldsymbol{M}-1$ characteristics diverge away from it, possibly spreading the error. Nevertheless, computations in a fixed mesh indicate that there is a definite improvement in the solution if a scheme with a higher order of accuracy is used. Because of insufficient analysis and numerical experimentation we cannot ascertain that the full order of accuracy $O\left((\Delta x)^{R}\right)$ is maintained at points sufficiently far removed from the shock, nor assess the damage, if any, to the order of accuracy. However, we feel that there is enough evidence of improvement in the solution generated by a fixed mesh computation to make the use of a hybrid scheme of the type (4.8) desirable.

The presence of a contact discontinuity in the solution is even more troublesome, because it is a discontinuity in a linearly degenerate field. Analysis of a system of linear equations in [20] shows that in this case, the accuracy in the whole domain of influence of the initial discontinuity is at most $O(\Delta x)$. The ACM causes the characteristic fields at a discontinuity to be artificially more convergent; a contact discontinuity is changed into a shock. Thus the ACM acts to decrease the propagation of the error from discontinuities.

7. Summary. In the previous sections of this paper we have shown how to modify standard difference schemes so that they self-adjust to the computation of discontinuities. The modification of the standard finite-difference scheme, which is a 
combination of hybridization and ACM, preserves the order of truncation error and yet allows sharp oscillation-free transitions of discontinuities. This hybrid ACM, being a modification of a standard scheme, is easy to implement in existing computer codes. In particular, it is easy to implement the hybrid ACM for the LW-type schemes (4.8) and (5.1): All one has to do is to add the adoptive artificial viscosity term $(1 / 8)\left[\theta_{j+1 / 2}\left(v_{j+1}-v_{j}\right)-\theta_{j-1 / 2}\left(v_{j}-v_{j-1}\right)\right]$ to the subroutine of a LW-type scheme and call a short independent ACM subroutine thereafter. The particular choice (4.8) for the LW-type schemes was primarily motivated by its generality and simplicity. It causes a reduction of the stability condition by at most $13.4 \%$; judging from comparison of the physical time in Figures 1-9 (shown in the upper left part of the figures), this limitation is not severe. Condition $(4.7 \mathrm{~b})$ can be somewhat relaxed by taking into account the variation of the automatic switch $\theta$. In dealing with a particular scheme, one can hybridize it with a nonoscillatory first order accurate scheme with a maximal stability condition, and by this prevent a possible cut of the permissible time step.

The additional computing time required for the use of the hybridization and the artificial compression depends strongly on the particular problem as well as on programming skill. We have experienced an average increase of $25 \%-30 \%$ with respect to the computing time of the unmodified LW-type schemes. In evaluating this additional investment in computing time one should take into account that the use of the hybrid ACM eliminates the need to add artificial viscosity terms.

The extension of the hybrid ACM to multidimensional problems is immediately available through a Strang-type dimensional operator splitting (see [24] and [7]). Both the hybridization technique (see [13]) and the ACM (see [8]) extend to several space dimensions without conceptual changes. A detailed discussion is deferred to a subsequent article.

Acknowledgement. This report was prepared as a result of work performed at the Courant Institute of Mathematical Sciences under U.S. Air Force Contract No. F44620-74-C-0062 and at NASA-Ames Research Center, Moffett Field, California, under Interchange NCA2-OR525-701.

I thank the members of the CFD branch at NASA-Ames, especially Bob MacCormack and Bob Warming, for many helpful discussions, as well as for their hospitality.

I thank Peter Lax, Sam Burstein and Heinz Kreiss for critically reading this manuscript; their remarks and suggestions were most helpful.

Appendix. Let $L$ be the hybrid scheme (4.8), $v^{n+1}=L v^{n}$,

$$
w_{j} \equiv(L v)_{j}=v_{j}-\frac{1}{2} \mu\left(v_{j+1}-v_{j-1}\right)+\frac{1}{2} \mu^{2}\left(v_{j+1}-2 v_{j}+v_{j-1}\right)
$$

$$
+\frac{1}{8}\left[\theta_{j+1 / 2}\left(v_{j+1}-v_{j}\right)-\theta_{j-1 / 2}\left(v_{j}-v_{j-1}\right)\right],
$$

where $\mu=\lambda A$ and $\theta_{j+1 / 2}$ is the automatic switch (4.12) with $\sigma(v)=v$ and $p=1$, i.e., $\Delta_{i+1 / 2} v=v_{i+1}-v_{i}$,

$$
\theta_{j+1 / 2}=\max \left(\hat{\theta}_{j}, \hat{\theta}_{j+1}\right)
$$




$$
\hat{\theta}_{j}=\hat{\theta}\left(\left|\Delta_{j-1 / 2} v\right|,\left|\Delta_{j+1 / 2} v\right|\right), \quad \hat{\theta}(x, y)=\left|\frac{x-y}{x+y}\right| .
$$

THEOREM. The hybrid scheme (A1) and (A2) is monotonicity preserving for $|\mu| \leqslant \sqrt{2} / 2$.

Proof. Let $v$ be any monotone mesh function, and let $\delta$ be the sign of its variation ( $\delta=1$ if $v$ is increasing, $\delta=-1$ if $v$ is decreasing). Subtracting (A.1) at $j=i$ from (A.1) at $j=i+1$, and then multiplying by $\delta$, we get after rearrangement

$$
\begin{aligned}
\delta \Delta_{i+1 / 2} w= & 1 / 2\left[(\mu+1 / 2)^{2}-1 / 4\left(1-\theta_{i-1 / 2}\right)\right]\left|\Delta_{i-1 / 2} v\right| \\
& +\left[3 / 4-\mu^{2}+1 / 4\left(1-\theta_{i+1 / 2}\right)\right]\left|\Delta_{i+1 / 2} v\right| \\
& +1 / 2\left[(\mu-1 / 2)^{2}-1 / 4\left(1-\theta_{i+3 / 2}\right)\right]\left|\Delta_{i+1 / 2} v\right| .
\end{aligned}
$$

It follows from (A.2a) that $\theta_{i-1 / 2} \geqslant \hat{\theta}_{i}$ and $\theta_{i+3 / 2} \geqslant \hat{\theta}_{i+1}$; therefore, the right-hand side of (A.3) is decreased upon replacing $\theta_{i-1 / 2}$ by $\hat{\theta}_{i}$ and $\theta_{i+3 / 2}$ by $\hat{\theta}_{i+1}$. Hence,

$$
\begin{aligned}
\delta \Delta_{i+1 / 2} w \geqslant & 1 / 2\left[(\mu+1 / 2)^{2}-1 / 4\left(1-\hat{\theta}_{i}\right)\right]\left|\Delta_{i-1 / 2} v\right| \\
& +\left[3 / 4-\mu^{2}+1 / 4\left(1-\theta_{i+1 / 2}\right)\right]\left|\Delta_{i+1 / 2} v\right| \\
& +\left[(\mu-1 / 2)^{2}-1 / 4\left(1-\hat{\theta}_{i+1}\right)\right]\left|\Delta_{i+3 / 2} v\right| \equiv F .
\end{aligned}
$$

Denoting $x=\left|\Delta_{i-1 / 2} v\right|, y=\left|\Delta_{i+1 / 2} v\right|$ and $z=\left|\Delta_{i+3 / 2} v\right|$, the right-hand side of (A.4), $F(\mu ; x, y, z)$ can be rewritten as

(A.5) $F(\mu ; x, y, z)=D(\mu ; x, y)+D(-\mu ; z, y)+y\left[1-\mu^{2}-1 / 4 \theta(x, y, z)\right]$, where $\theta(x, y, z)=\max (\hat{\theta}(x, y), \hat{\theta}(y, z))$ and

$$
D(\mu ; x, y)=1 / 2\left[(\mu+1 / 2)^{2}+1 / 4(\hat{\theta}(x, y)-1)\right] x .
$$

We prove the theorem by showing that

$$
F(\mu ; x, y, z) \geqslant 0 \text { for }|\mu| \leqslant \sqrt{2} / 2 \text { and all nonnegative } x, y, z .
$$

Since $F(\mu ; x, y, z)=F(-\mu ; z, y, x)$ it is sufficient to show that $F(\mu ; x, y, z) \geqslant 0$ for $0 \leqslant \mu \leqslant \sqrt{2} / 2$. Evaluation of $(d / d z)[z(\hat{\theta}(y, z)-1)]$, where $\hat{\theta}$ is defined by (A.2b), yields

$$
\frac{d}{d z}[z(\hat{\theta}(y, z)-1)]= \begin{cases}-\frac{2 y^{2}}{(z+y)^{2}} & \text { for } z>y, \\ -\frac{2 z(z+2 y)}{(z+y)^{2}} & \text { for } z \leqslant y .\end{cases}
$$

Thus, $(d / d z)[z(\hat{\theta}(y, z)-1)] \leqslant 0$ for all $z \geqslant 0$ and $y \geqslant 0$; consequently,

$$
z(\hat{\theta}(y, z)-1) \geqslant \lim _{z \rightarrow \infty} z(\hat{\theta}(y, z)-1)=\lim _{z \rightarrow \infty} \frac{-2 y z}{y+z}=-2 y .
$$

Using (A.8) in $D(-\mu ; z, y)$, we get

$$
D(-\mu ; z, y) \geqslant 1 / 2(\mu-1 / 2)^{2} z-1 / 4 y .
$$


Using the above inequality in (A.5), we get

$$
\begin{aligned}
F(\mu ; x, y, z) \geqslant & D(\mu ; x, y)+1 / 2(\mu-1 / 2)^{2} z \\
& +\left[1 / 2-\mu^{2}+1 / 4(1-\theta(x, y, z))\right] y ;
\end{aligned}
$$

but $D(\mu ; x, y) \geqslant 0$ for $\mu \geqslant 0$ and the last term in the right-hand side of (A.9) is nonnegative for $|\mu| \leqslant \sqrt{2} / 2$. Hence the right-hand side of (A.9) is nonnegative for $0 \leqslant$ $\mu \leqslant \sqrt{2} / 2$. This completes the proof.

Remark. (A.4) for $|\mu|=\sqrt{2} / 2$ is a strict inequality except for the trivial case $x=y=z=0$; thus, the theorem holds beyond $|\mu|=\sqrt{2} / 2$.

Courant Institute of Mathematical Sciences

New York University

251 Mercer Street

New York, New York 10012

1. J. P. BORIS \& D. L. BOOK, "Flux corrected transport. I. SHASTA, a fluid transport algorithm that works," J. Computational Phys., v. 11, 1973, pp. 38-69.

2. S. Z. BURSTEIN, "Finite difference calculations for hydrodynamic flows containing discontinuities," J. Computational Phys., v. 1, 1966, pp. 198-222.

3. A. J. CHORIN, "Random choice solution of hyperbolic systems," J. Computational Phys., v. 22, 1976, pp. 517-533.

4. R. COURANT \& K. O. FRIEDRICHS, Supersonic Flow and Shock Waves, InterscienceWiley, New York, 1948.

5. S. K. GODUNOV, "Finite difference methods for numerical computations of discontinuous solutions of equations of fluid dynamics," Mat. Sb., v. 47, 1959, pp. 271-295. (Russian)

6. M. GOLDBERG \& S. ABARBANEL, "Stable approximations for hyperbolic systems with moving internal boundary conditions," Math. Comp., v. 28, 1974, pp. 413-447.

7. D. GOTTLIEB, "Strang-type difference schemes for multi-dimensional problems," SIAM J. Numer. Anal., v. 9, 1972, pp. 650-661.

8. A. HARTEN, The Method of Artificial Compression: I. Shocks and Contract Discontinuities, AEC Research \& Develop. Rep. COO-3077-50, Courant Inst., New York Univ., June 1974.

9. A. HARTEN, "The artificial compression method for computation of shocks and contact discontinuities: I. Single conservation laws," Comm. Pure Appl. Math. (To appear; also to appear as an ICASE Report.)

10. A. HARTEN, "The artificial compression method for computation of shocks and contact discontinuities: II. Systems of conservation laws." (In preparation.)

11. A. HARTEN, J. M. HYMAN \& P. D. LAX, "On finite-difference approximations and entropy conditions for shocks," (with Appendix by B. Keyfitz), Comm. Pure Appl. Math., v. 29, 1976, pp. 297-322.

12. A. HARTEN \& G. ZWAS, "Switched numerical Shuman filters for shock calculations," J. Engrg. Math., v. 6, 1972, pp. 207-216.

13. A. HARTEN \& G. ZWAS, "Self-adjusting hybrid schemes for shock computations," J. Computational Phys., v. 6, 1972, pp. 568-583.

14. G. JENNINGS, “Discrete shocks," Comm. Pure Appl. Math., v. 27, 1974, pp. 25-37.

15. A. LAPIDUS, "A detached shock calculation by second-order finite differences," $J$. Computational Phys., v. 2, 1967, pp. 154-177.

16. P. D. LAX, "Hyperbolic systems of conservation laws. II," Comm. Pure Appl. Math., v.10, 1957 , pp. 537-566.

17. P. D. LAX \& B. WENDROFF, "Systems of conservation laws," Comm. Pure Appl. Math., v. 13, 1960, pp. 217-237.

18. T. P. LIU, “The entropy condition and the admissibility of shocks," J. Math. Anal. Appl., v. 53, 1976, pp. 78-88.

19. R. W. MacCORMACK, Numerical Solution of the Interaction of a Shock Wave with a Laminar Boundary Layer (Proc. 2nd Internat. Conf. on Numerical Methods in Fluid Dynamics, M. Holt, Editor), Lecture Notes in Phys., v. 8, Springer-Verlag, New York, 1970, pp. 151-163. 
20. A. MAJDA \& S. OSHER, "Propagation of errors into regions of smoothness for accurate difference approximations to hyperbolic equations," Comm. Pure Appl. Math. (To appear.)

21. O. A. OLEINIK, "Discontinuous solutions of nonlinear differential equations," Uspehi Mat. Nauk, v. 12, 1957, pp. 3-73; English transl., Amer. Math. Soc. Transl. (2), v. 26, 1963, pp. $95-172$.

22. S. A. ORSZAG \& L. W. JAYNE, “Local errors of difference approximations to hyperbolic equations," J. Computational Phys., v. 14, 1974, pp. 93-103.

23. R. D. RICHTMYER \& K. W. MORTON, Difference Methods for Initial Value Problems, 2nd ed., Interscience-Wiley, New York, 1967.

24. G. STRANG, "On the construction and comparison of difference schemes," SIAM J。 Numer. Anal., v. 5, 1968, pp. 506-517.

25. H. U. THOMMEN, "Numerical integration of the Navier-Stokes equations," $Z$. Angew. Math。Phys., v. 17, 1966, pp. 369-384.

26. B. VAN LEER, "Towards the ultimate conservative difference scheme. II. Monotonicity and conservation combined in a second order scheme," J. Computational Phys., v. 14, 1974, pp. $361-370$.

27. J. von NEUMANN \& R. D. RICHTMYER, "A method for the numerical calculation of hydrodynamic shocks," J. Appl. Phys., v. 21, 1950, pp. 232-237.

28. N. N. YANENKO \& Yu. I. SHOKIN, "First differential approximation method and approximate viscosity of difference schemes," Phys. Fluids, suppl. II, v. 12, 1969, pp. II-28-II-33. 